\title{
Euthanasia in Wildlife Rehabilitation and Specific Considerations for Pelagic and Diving Bird Rehabilitation
}

JANUARY O. BILL

International Bird Rescue Research Center

FAIRFIELD, CALIFORNIA

\section{INTRODUCTION}

It is the goal and responsibility of wildlife rehabilitators to provide the best achievable care to the animals that are brought to them for help. Because of the nature of wildlife rehabilitation, by the time the rehabilitator receives the animal, its injuries and/or illness have often progressed to a state beyond a point that will enable the animal to survive when returned to the wild. If an animal cannot be returned to a normal life in the wild, the only options rehabilitators have for a non-releasable animal in their care is life in captivity or euthanasia (Miller 1993). Just as it is vital to have the skills and knowledge to successfully treat a species of wildlife, it is equally important to have the skills to evaluate the animal for euthanasia on intake. Euthanasia is often the only viable option to humanely end an animal's pain and prevent further suffering. Therefore, euthanasia decisions based on physiological evidence and well-reasoned judgment lead to more humane treatment of wildlife in rehabilitation.

The objective of this paper is to emphasize the importance of this aspect of wildlife rehabilitation, to provide basic euthanasia guidelines, and to outline a set of specific considerations for pelagic and diving bird rehabilitation used at International Bird Rescue Research Center (IBRRC).

It can often be difficult to make a choice regarding euthanasia. All of the decisions that wildlife rehabilitators make are based on facts determined during intake and treatment, and the ethics of the individual rehabilitator and his or her organization. The more facts a rehabilitator can accumulate while maintaining strong ethics, the easier it becomes to make good decisions (Miller 2001). When evaluating an animal

January O. Bill received her BS with a major in wildlife rehabilitation from Humboldt State University. She is currently working for the International Bird Rescue Research Center as an oil spill response team member, and as the Training Coordinator. for euthanasia, it is vital that one look at the overall health of the individual animal and not just their primary injury or disease. By doing so, secondary issues may be identified that could affect the probability of the animal returning to a state of health where it could be successfully returned to the wild. Consulting with wildlife veterinarians, fellow rehabilitators, and specific guidelines, are key elements for the humane treatment of wildlife and for the successful progression of wildlife rehabilitation as a whole.

Euthanasia decisions should be based on: physical exam findings, secondary health concerns, diagnostic results, and consultation with your veterinarian. There are basic guidelines that apply to all wildlife rehabilitators and wildlife veterinarians when evaluating wildlife for euthanasia. White (1993) outlines several questions rehabilitators should consider when evaluating animals for euthanasia:

- Is the injury repairable or disease curable?

- What is the degree of suffering; can it be alleviated?

- What is the level of care that can be offered?

- Can one provide appropriate care for that particular species given the current caseload?

- What will be the quality of life for the animal during and after rehabilitation?

- Does the animal carry a disease that could be transmitted to the wild population?

All of White's (1993) questions taken collectively help determine an animal's probability of surviving the rehabilitation process. Often rehabilitators focus on the first question: Is the injury repairable or disease curable? Each of these questions, however, should be given equal weight when evaluating an animal for euthanasia, because each answer is critical to ethical and successful wildlife rehabilitation.

While the release of animals after rehabilitation and their long-term survival are always the ultimate 
goals of any rehabilitation program, euthanasia cases should not be viewed as failures. Nearly all wildlife brought to rehabilitators are compromised to a point where they were unable to survive in their environment without human intervention. Many of these animals are dying or near death with traumatic injuries, debilitating diseases, and/or exposure to contaminants. Many of these cases will not survive the rehabilitation process and the only humane treatment is euthanasia. Consequently, educated euthanasia decisions should not be viewed as failures, but rather as the only appropriate measure to alleviate an animal's pain and to prevent further suffering. And so, understanding when to euthanize and recognizing its important role in wildlife rehabilitation leads to more humane treatment of wildlife.

In order to make an educated and confident euthanasia decision, the wildlife rehabilitator needs to be familiar with the species one is working with, both behaviorally and physiologically. This familiarity is important because different species recover differently from injury and disease in a captive setting. When evaluating an animal that is unfamiliar, it is important to consult wildlife veterinarians or wildlife rehabilitators that have experience with that specific species. Networking with other rehabilitators can help provide information that can significantly influence the choices made. Networking also creates the option to transport the animal to another organization that has the resources and experience to better rehabilitate that species.

The basic euthanasia guidelines mentioned above should be supplemented with a set of tailored considerations that are specific to one's own organization and the species that is cared for. Having this set of specific criteria for the organization helps to prevent unintentional inhumane treatment of wildlife and allows the focus to be on the animals that have a higher probability of survival. Guidelines can be developed using evidence from prior cases with similar clinical symptoms, the organization's capabilities, and the resources available.

\section{SPECIFIC CONSIDERATIONS FOR PELAGIC AND DIVING BIRDS}

Euthanasia criteria have been developed for pelagic and diving birds treated at International Bird Rescue Research Center in Fairfield and San Pedro, California. Specializing in aquatic bird rehabilitation, IBRRC has 34 years of experience rescuing and rehabilitating injured, oiled, and orphaned wildlife. Since 2000, through a unique partnership with the Oiled Wildlife Care Network, IBRRC has had the opportu- nity to work in facilities that were designed specifically for oiled aquatic bird rehabilitation. Because pelagic birds are extremely susceptible to severe secondary health complications as a result of being in captivity, guidelines based on physiological evidence have been essential for evaluating these species' chances of being successfully rehabilitated at these facilities.

\section{Why PELAgIC AND DiVING BIRD REHABILITATION IS SPECIALIZED}

Rehabilitation of pelagic and diving birds is specialized because these types of birds live most of their lives entirely on water. The time to successfully rehabilitate these birds before life-threatening secondary complications begin to develop is extremely short compared to other species of wildlife.

Pelagic and diving birds have bodies adapted to live in water. Therefore, because of the vast time they spend swimming, the pelvic limbs are far caudal on the body, so these birds lack the ability to stand on land or to maintain an upright posture while bearing weight on the caudal surface of the whole of the lower limb below the hock (Tully et al 2000). When these birds inhabit a non-water environment, abnormal weight and pressure is applied to their bodies. These contact areas can quickly develop feather loss and feather damage, which progress to pressure lesions.

Their limited exposure to land-based diseases increases their susceptibility when on land and in captive settings. These birds are easily stressed which can exacerbate the complications mentioned above. They are frequently victims of contamination, which causes waterproofing and thermoregulation problems.

Being able to recognize these issues on intake and to make decisions regarding euthanasia based on these conditions as well as the overall health of the bird prevents the unnecessary prolonging of a rehabilitation process that will likely end unsuccessfully. Preventative measures exist that can be taken to help minimize and reduce the occurrence of these secondary complications that can end in death. However, time is the limiting factor, and very often birds arrive with these existing complications. Knowing when to treat and when to euthanize are critical and crucial decisions.

Most of these life-threatening conditions may appear superficial to people who are inexperienced in rehabilitating these species, which is why it is important to network with other organizations to gather information on animals unfamiliar to one's organization. Being able to recognize and understand the severity of these secondary complications is vital for euthanasia evaluations and the successful rehabilitation of pelagic and diving birds. 


\section{PELAgIC AND DiVING BIRD EUTHANASIA CONSIDERATIONS}

Wildlife rehabilitation is a fast growing field. As new techniques are developed and wildlife medicine evolves, some of the injuries and diseases that currently result in poor prognosis may be treatable. In addition to general euthanasia criteria, the considerations below are specific to pelagic and diving birds, and based on past cases of wildlife rehabilitation at IBRRC.

Multiple Strikes. Upon intake, if a seabird has several substantial problems, the likelihood of that bird living through the rehabilitation process without developing life-threatening secondary complications is very low. If the bird has multiple issues affecting its condition, the probability of the bird completely recovering from its initial problems without succumbing to secondary complications should be evaluated. In short, the number and severity of ailments on intake should be carefully assessed and the time frame for rehabilitation considered.

Loss of Waterproofing. Pelagic and diving birds cannot live without feathers that keep them waterproof. Once a bird loses its waterproofing, water is able to reach its skin, creating a situation where the bird becomes hypothermic and, in time, will lose buoyancy and drown (Goodfriend 1997). If the bird is unable to remain in the water, it becomes susceptible to secondary complications. Several reasons exist for loss of waterproofing; for instance, changes in the normal structure of a feather (by becoming broken, soiled or otherwise damaged) will reduce the water-resistant properties of the feather and will allow water to penetrate this barrier (Thorne 1986). The health of these birds will quickly deteriorate if they cannot be housed in a water-based environment, so it is imperative that the bird quickly become waterproof or at least be able to be housed in water that is temperature controlled. The goal is to have pelagic and diving birds that are stable and uncontaminated in a water environment within 24 hours of arrival.

One must consider several factors when determining the need for euthanasia with regards to loss of waterproofing. Some considerations for euthanasia could be poor feather quality, permanent scar tissue with no feather re-growth, and fol- liculitis. Clinical presentations of poor waterproofing include: rising up out of the water, reluctance to be in water, excessive preening, fluffed feathers, abnormal buoyancy for the species, shivering, missing or parted feathers, inability to thermoregulate, and being wet to the skin.

Feather Quality. The plumage of the bird should be examined for missing and damaged feathers and overall feather condition. Common reasons for degraded feather quality are contaminants, feather structure damage, and missing feathers caused by injury or disease. Depending on the severity and condition, some of these issues can be resolved with time.

Questions that should answered: Can the condition be treated? Will the condition allow the bird to be housed in water? How long will the treatment take? The time of the year should also be considered when birds present with feathers that are damaged or missing. The bird may be in the process of molting, which could explain the poor feather condition. The length and pattern of the molt must be fully understood for the species in question. Another reason for loss of waterproofing due to poor feather quality is folliculitis. Folliculitis is a condition frequently seen in common murres (Uria aalge) (Figure 1), and is a viral or bacterial infection in the feather follicle causing feather loss and feather structure damage that usually results in loss of waterproofing. Clinical signs include: patches of broken or stripped contour feath-

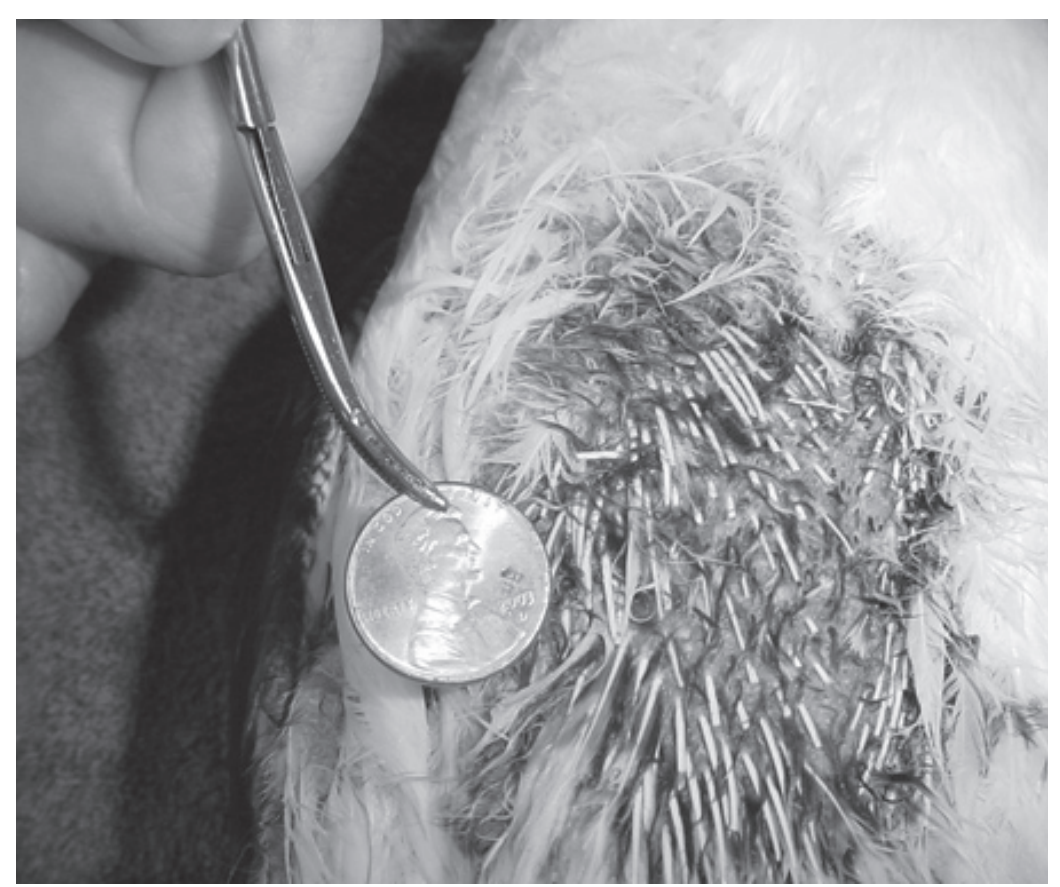

Figure 1. Folliculitis patch on a common murre. Clinical signs that are visible include stripped coverts, red irritated skin, yellow exudates at base of feather shaft, and missing down. 


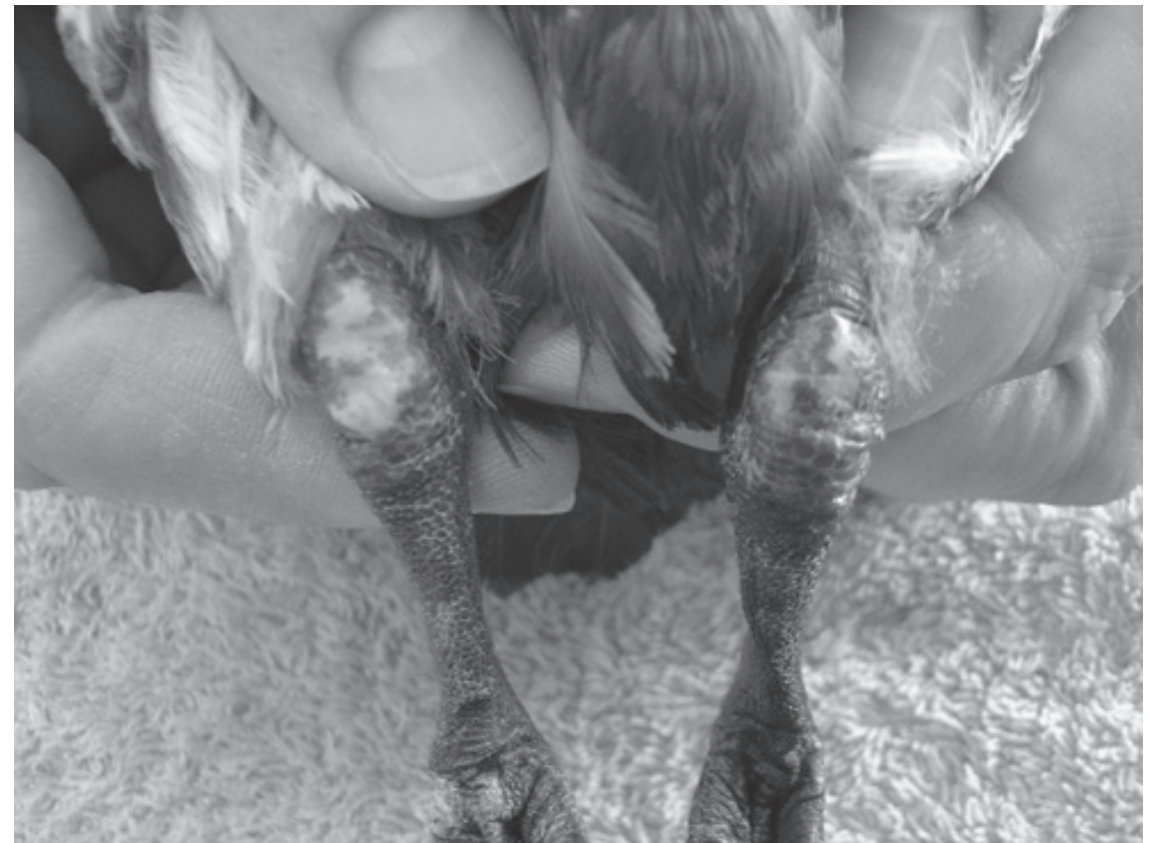

Figure 2. Common murre with severe hock pressure lesions. Clinical signs visible in this photograph are the swollen, puffy appearance of both the hocks and feet, and the skin discoloration.

ers, absence of down feathers, irritated red skin, yellow crusty exudates around feather follicles. Treatment with systemic antibiotics may be attempted with small dime-sized patches (approximately $1 \mathrm{~cm}^{2}$ ). Birds that are not able to remain waterproof and have a patch of folliculitis that is greater than the size of a dime with no signs of down re-growth should be evaluated for euthanasia.

\section{Time Housed Out of Water.}

When housed out of the water on a land-based environment, physical secondary complications will begin to develop, often within as little as 24 hours. Injured, diseased, or contaminated birds that are required to be housed in a non-water enclosure for an extended period of time will likely succumb to secondary complications. A question that should be asked is: How long will the bird have to be housed out of water until it can recover to a state where it can be returned to water? If this time is greater than five days, the chance of successful rehabilitation is poor (Unpublished IBRRC data).

Pressure Sores. Pressure sores are soft tissue lesions caused by abnormal weight placement. Often in pelagic and diving birds they start developing once the birds have beached themselves. If birds with pressure sores are not managed immediately in a water environment, the lesions will often lead to exposed bone, decreased mobility of affected limbs, systemic infection, and eventually death.

Common areas where pressure sores develop are the hocks (Figure 2) and feet (Figure 3). The bone in the affected area can quickly become infected, and infection involving bone (osteomyelitis) is generally difficult to treat. Osteomyelitis often requires long-term antibiotic treatment and surgery to remove necrotic tissue (Ritchie et al 1994). Clinical signs often include a combination of the following: a swollen or puffy appearance to the affected area, which may be abnormally warm to the touch, localized abnormal skin coloring, red irritated area(s) of skin, and open lesions over joints. Past cases have shown that signs of hock and foot lesions associated with loss of mobility, bone, and joint infections, exposed tendons, and/or systemic infection are grounds for euthanasia consideration.

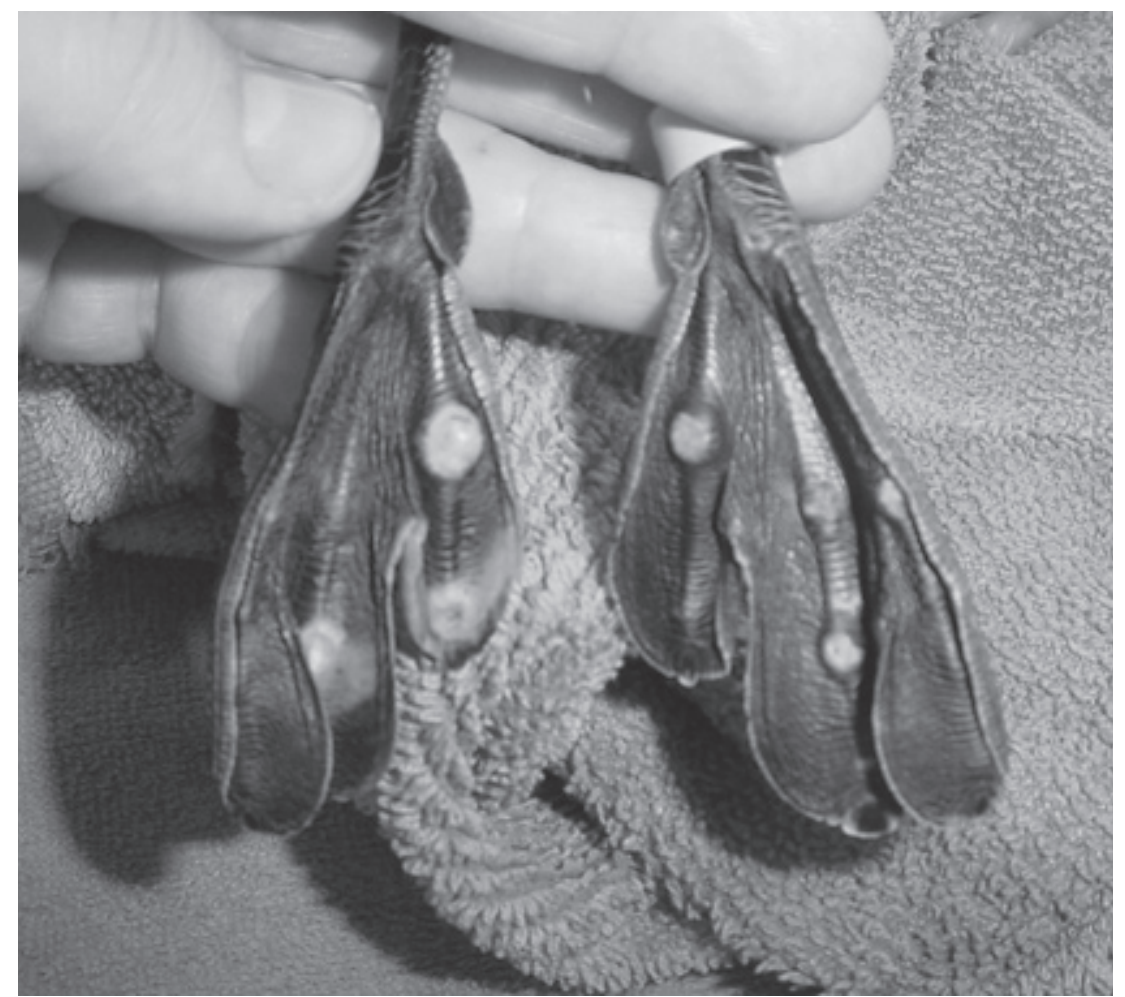

Figure 3. Pressure lesions at the toe joints of a pied-billed grebe (Podilymbus podiceps). 
Pressure sores along the keel (Figure 4) are another area of concern. This is particularly crucial with birds that have been beached for any length of time. The apex of the keel consists of thin skin covering bone. Pressure sores along the apex quickly develop and progress when pelagic birds are out of water. The sore rapidly progresses to a thickening of the skin, and then to an open lesion that exposes the sternum (Goodfriend 1997). Clinical signs to look for include: damaged covert feathers along the keel, missing down, red irritated skin, wet to skin areas along the keel, skin adhered to the keel (no longer sliding over the bone), and visible lesions. Birds with any combination of a loss of waterproofing along keel, exposed bone, adhered skin, and infection have a very poor prognosis.

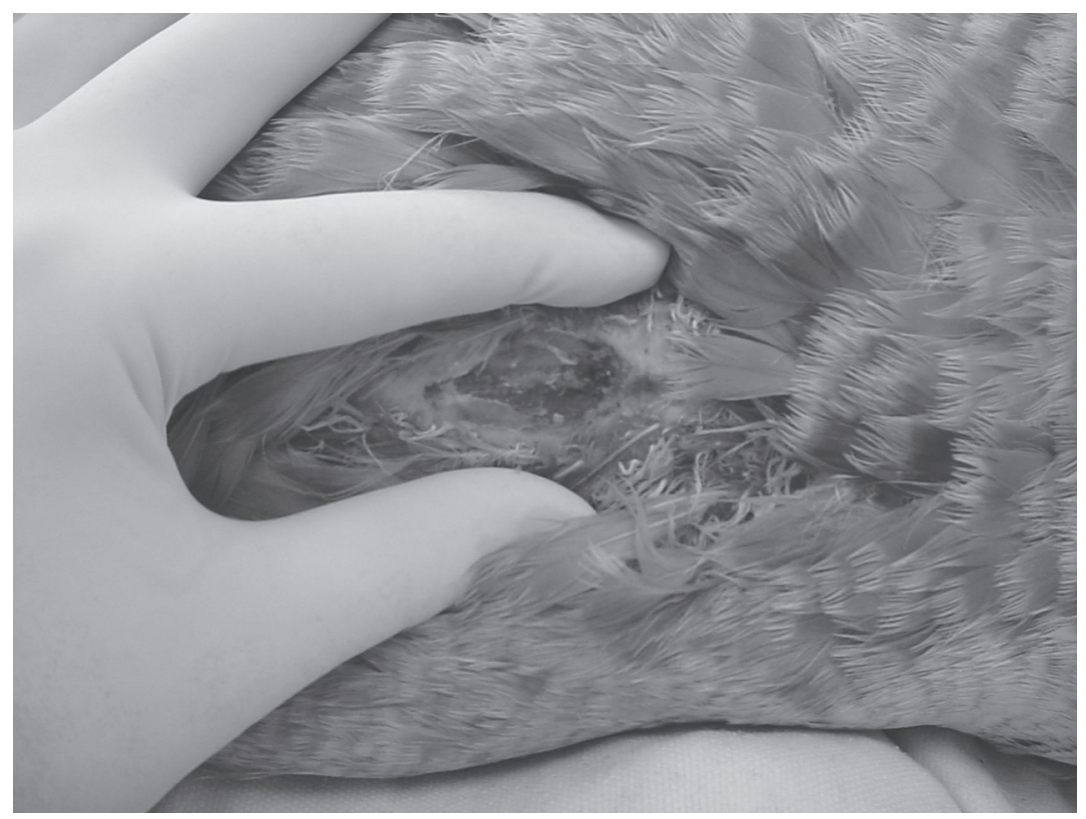

Figure 4. Severe keel lesion that is exposing bone.

Respiratory Infections. Seabirds that are immunocompromised from injury, disease, antibiotics, steroids, and/or stress are highly susceptible to aspergillosis, a land-based fungal infection caused by Aspergillus fumigatus. Treatment of aspergillosis is rarely effective once clinical signs are established (Tully et al 2000). While it is difficult to diagnose, clinical presentations can include: dyspnea, open-mouth breathing, low packed cell volume with an increasing total protein, increasing white blood cell count, unexplained weight loss, abnormal lung sounds, depressed attitude, and/ or sudden death.

Pneumonia is the inflammation of one or both lungs, usually caused by a bacterial or viral infection, and it often leads to a poor outcome in pelagic and diving bird rehabilitation. Clinical signs often include: wet cough, gurgling sounds heard in lungs, lethargy and weight loss, open-mouth breathing, and/or dyspnea. Treatment usually includes a course of antibiotics, which may affect the natural microbial flora, consequently making birds more susceptible to aspergillosis.

Fractures. The treatment of fractures varies depending on the species and the type and location of the fracture. Most leg and wing fractures are not treated in pelagic and diving birds because perfect range of motion and mobility are needed for these species to survive in the wild, and fractures frequently result in altered range of motion. These birds need to be in optimal physical condition to dive for food and to escape predators. Treatment usually means the birds need to be out of water while the bone is healing, thus increasing their susceptibility to the secondary problems mentioned above. Euthanasia should be considered for any pelagic or diving birds with fractures requiring pinning and/or long-term treatment.

Blood Values. Once pelagic and diving birds have beached themselves on land, they are unable to feed or drink. Most of these birds arrive at IBRRC with some level of dehydration, starvation, and anemia. It is important to run basic blood work to gain insight into the severity of these conditions. Packed cell volume (PCV), total solid (total protein), and buffy coat values should be determined. Normal ranges of avian blood values taken from Campbell (1995) are as follows:

- Packed cell volume: 35 to 55 percent

- Buffy coat: less than 2.0 percent

- Total protein: 3.5 to $5.5 \mathrm{~g} / \mathrm{dL}$

Birds that have packed cell volumes of less than 15 percent and total proteins of less than $1 \mathrm{~g} / \mathrm{dl}$ have a very poor prognosis.

In addition to the initial blood values, the dehydration level of the bird must be considered. With moderate to severe dehydration, the PCV and total protein can be falsely elevated. A PCV greater than 55 percent is suggestive of dehydration (Ritchie et al 1994). If blood work values are being used to determine the necessity of euthanasia, tests should be repeated to avoid a misdiagnosis. 


\section{SUMMARY}

Being able to accurately assess an animal's chance of being rehabilitated and released, and to emotionally separate ourselves from a false sense of responsibility are vital skills a wildlife rehabilitator should strive to master. Advanced rehabilitators regard the decision of euthanasia as a duty. They understand the need for euthanasia and the necessity of placing the interest of the animal above their personal needs (McKeever 1993). Having a set of specific euthanasia guidelines to supplement general euthanasia criteria will help rehabilitators make more informed euthanasia decisions and will ultimately result in more humane treatment of wildlife.

Dr. Miller (1993) summarizes the significance of euthanasia best: "The animal with terminal illness or fatal injury deserves the best treatment we can give it. We have no right prolonging that animal's pain or discomfort by allowing the animal to die without our assistance."

\section{LITERATURE CITED}

Campbell, T. 1995. Avian Hematology and Cytology.

Iowa State University Press: Ames, IA. Pp. 3-19.

Goodfriend, D. 1997. Considerations in Seabird

Rehabilitation. Pp. 21-29 in International Wildlife

Rehabilitation Council Conference Proceedings.

McKeever, K. 1993. Quality of Life. NWRA Newsline.

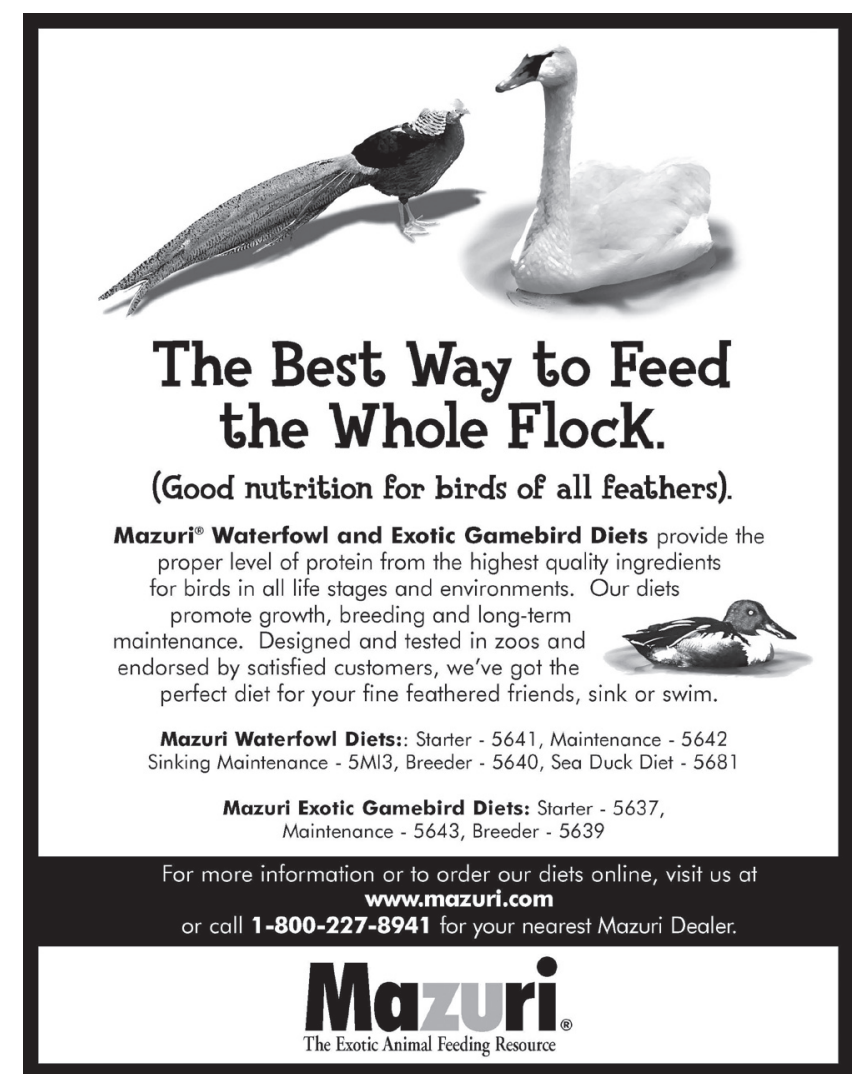

National Wildlife Rehabilitators Association: St. Cloud, MN. 11(3):6.

Miller, E. 1993. Euthanasia, the Other Release. NWRA Newsline. National Wildlife Rehabilitators Association: St. Cloud, MN. 11(3):10-11.

Miller, E. 2001. Ethics in Wildlife Rehabilitation. Pp. 151-160 in Wildlife Rehabilitation (D. Ludwig, ed.), Vol. 19. National Wildlife Rehabilitators Association: St. Cloud, MN.

Ritchie, B., G. Harrison, and L. Harrison. 1994. Avian Medicine: Principles and Application. Wingers Publishing, Inc: Lake Worth, FL. P. 967.

Thorne, K. 1986. Is Your Bird Waterproof? Wildlife Journal. International Wildlife Rehabilitation Council: Walnut Creek, CA. 9(2):7-10.

Tully, T., M. Lawton, and G. Dorrestein. 2000. Avian Medicine. Reed Educational and Professional Publishing, Ltd: Woburn, MA. Pp. 339-363.

White, J. 1993. Current Guidelines for Euthanasia in Wildlife Rehabilitation. Journal of Wildlife Rehabilitation. 16(3):19-23.

\section{ACKNOWLEDGMENTS}

Information presented for pelagic and diving bird euthanasia considerations were developed by staff and volunteers at IBRRC. Thank you to Kenneth M. Griggs, Marie Travers, and Michelle Bellizzi for reviewing and commenting on this paper.

\section{NOTICE TO REAdERS}

An error occurred in the Wildlife Rehabilitation Bulletin, Volume 23, Number 1, Spring/Summer 2005, pages 4146. Infectious Diseases of North American Black Bears (Ursus americanus) by Dawn Zimmerman, DVM, MS, and Mark A. Mitchell, DVM, MS, PhD, is a two part series with Part 1 on viral and bacterial pathogens and Part 2 on parasites. Part 1 was inadvertently printed twice. Part 2 will appear in Volume 24, Number 1, Spring/ Summer 2006. The editors extend their sincere apologies to Dr. Zimmerman, Dr. Mitchell, and our readers for this error. 